\title{
ACCIONES DIDÁCTICAS EN EL DESARROLLO DE UN RECORRIDO DE ESTUDIO Y DE INVESTIGACIÓN QUE INVOLUCRA A LA MATEMÁTICA Y A LA FÍSICA EN LA ESCUELA SECUNDARIA
}

\author{
DIDACTIC ACTIONS IN THE DEVELOPMENT OF A STUDY AND RESEARCH \\ PATH THAT INVOLVES MATHEMATICS AND PHYSICS IN SECONDARY \\ SCHOOL
}

\author{
María Paz Gazzola (*) \\ María Rita Otero \\ Viviana Carolina Llanos \\ Núcleo de Investigación en Ciencia y Tecnología \\ Universidad Nacional del Centro de la Provincia de Buenos Aires \\ Argentina
}

\section{Resumen}

En este trabajo se presentan resultados sobre el desarrollo de un Recorrido de Estudio y de Investigación (REI) que involucra a la Matemática y a la Física, a partir de las acciones o gestos didácticos propuestos por la Teoría Antropológica de lo Didáctico (TAD) como propios de un REI, llamados dialécticas (Chevallard, 2013a). El REl fue implementado en cinco cursos de Matemática en escuelas secundarias con $\mathrm{N}=116$ estudiantes en total. Se emplean técnicas cualitativas de análisis y metaanálisis de datos para construir una categorización inductiva conforme al uso de técnicas cuantitativas de estadística multivariada. Los resultados muestran las dificultades del desarrollo de estos gestos en el aula y permiten interpretar la existencia de un funcionamiento conjunto de ciertas dialécticas, puesto que el aumento de frecuencia de ocurrencia de algunas se corresponde con el aumento de las otras.

Palabras clave: Escuela secundaria; TAD; REl; dialécticas; análisis estadístico multivariado.

$\left({ }^{*}\right)$ Autor para correspondencia: María Paz Gazzola

Universidad Nacional del Centro de la Provincia de Buenos Aires, Argentina Núcleo de Investigación en Ciencia y Tecnología

Pinto 399, Tandil, Buenos Aires, Argentina Correo de contacto:

mpgazzola@exa.unicen.edu.ar

C2010, Perspectiva Educacional Http://www.perspectivaeducacional.cl

RECIBIDO: 07.09.2019

ACEPTADO: 23.12 .2019

DOI: 10.4151/07189729-Vol.59-Iss.1-Art.1006
This paper presents results on the development of a Study and Research Path (SRP) that involves mathematics and physics, from the didactic actions or gestures proposed by the Anthropological Theory of the Didactic (ATD) as typical of a SRP, called dialectics (Chevallard, 2013a). The SRP was implemented in five secondary school math courses with $\mathrm{N}=116$ students in total. Qualitative techniques for data analysis and meta-analysis are used to construct an inductive categorization according to the use of multivariate quantitative techniques. The results show the difficulties of the development of these gestures in the classroom and allow to interpret the existence of a joint operation of certain dialectics, since the increase in the frequency of occurrence of some corresponds to the increase in the others.

Keywords: Secondary school; ATD; SRP; dialectics; multivariate statistical analysis. 


\section{INTRODUCCIÓN Y ANTECEDENTES}

Los Recorridos de Estudio y de Investigación (REI) son dispositivos didácticos propuestos por la Teoría Antropológica de lo Didáctico (TAD) para recuperar el sentido y las razones de ser de los saberes que se estudian en la escuela. Se parte del principio según el cual las preguntas son esenciales en la generación del saber (Chevallard, 2007, 2009), por lo tanto, el desarrollo de un REI requiere llevar a cabo ciertas acciones didácticas, llamadas dialécticas, como requisito para enseñar a partir del estudio y de la investigación. Estas acciones didácticas son ajenas a la forma de enseñanza denominada tradicional y no son responsabilidad exclusiva del profesor, por el contrario, se producen en el hacer individual y colectivo de una clase (Otero, Llanos, Parra \& Sureda, 2014; Parra \& Otero, 2018).

La investigación relativa a cómo enseñar mediante REI es reciente. En el artículo de Parra y Otero (2018) se realiza una compilación exhaustiva de 41 trabajos sobre los REI, encontrándose que solo 23 de ellos presentan el diseño, análisis y/o evaluación de recorridos implementados al menos una vez en algún nivel educativo. En el nivel secundario, pueden mencionarse los trabajos de Ruiz, Bosch y Gascón (2007), Gazzola, Llanos y Otero (2013), Llanos y Otero (2013), Parra, Otero y Fanaro (2013), Donvito, Otero y Sureda (2014), Gazzola, Otero, Llanos y Arlego (2015), Llanos, Otero y Gazzola (2017) y Sala, Barquero y Font (2018). Así mismo, Parra y Otero (2018) señalan que son muy pocas las investigaciones que se han ocupado de analizar el funcionamiento de las dialécticas en un REI. En los trabajos de Barquero (2009) y Costa, Arlego y Otero (2014) solo se realiza un análisis descriptivo del funcionamiento de las dialécticas haciendo referencia a los resultados de implementaciones en el nivel universitario; mientras que en los trabajos de Parra y Otero $(2017,2018)$ y Salgado, Otero y Parra (2017) se han utilizado las dialécticas como principal instrumento de análisis. Parra y Otero (2017) generaron un conjunto de indicadores didáctico-matemáticos de cada dialéctica a partir de los resultados de dos implementaciones en el nivel secundario; y por su parte, Salgado et al. (2017) ampliaron y caracterizaron las dialécticas en un REI para el nivel universitario.

En este trabajo, nos proponemos analizar el funcionamiento de un REI que involucra a la Matemática y a la Física en la escuela secundaria (Gazzola, 2018; Otero, Gazzola, Llanos \& Arlego, 2016) utilizando el constructo teórico dialéctica (Chevallard, 2013b). Se pretende responder las preguntas: ¿Cómo se puede establecer, a partir de los resultados de las implementaciones, la presencia de las dialécticas propias del desarrollo de un REI? ¿Cuáles gestos del estudio y de la investigación ocurren más frecuentemente y cuáles son más difíciles de concretar? Para ello, se usan técnicas de análisis y metaanálisis de datos cualitativos (Otero, Moreira \& Greca, 2002) y se realiza una categorización inductiva orientada al uso de técnicas cuantitativas multivariadas 
(Benzécri, 1980; Lebart, Morineau \& Fenelon, 1985) que se aplican mediante el software SPAD8. $1^{\circledR}$. Este estudio permite identificar las dialécticas y poner en evidencia las dificultades, condiciones y restricciones que pesan sobre su funcionamiento durante el desarrollo del REI, tal como presentaremos en las secciones siguientes.

\section{LOS REI Y LAS DIALÉCTICAS QUE PERMITEN GESTIONARLO}

Los REI tienen por objetivo introducir en las instituciones escolares una nueva epistemología que otorgue sentido y funcionalidad al estudio de la Matemática (Chevallard, 2009, 2013b; Otero et al., 2013). El inicio de un REl es una pregunta "en sentido fuerte", cuya respuesta no es inmediata y requiere de un largo proceso de construcción. En la TAD, una pregunta con estas características se denomina generatriz y se expresa utilizando la letra $Q$. En una enseñanza por REI, el profesor debe proponer la pregunta $Q$ y el grupo de clase debe producir una respuesta posible. Para eso, se requiere elaborar un medio apropiado, en términos de los saberes necesarios y los instrumentos requeridos. Recurriendo a una metáfora ecológica, el medio didáctico, expresado con la letra $M$, puede entenderse como un ambiente apropiado para elaborar las respuestas (Otero et al., 2013). Este medio está formado por tres elementos:

- $\quad$ Preguntas derivadas, que son aquellas que se desprenden de la pregunta cuestión Q;

- Respuestas "hechas", que también reciben el nombre de "etiquetadas", porque son reconocidas como tales en una institución y que se pueden encontrar en un libro, en un sitio web, en un applet o simulación, etc.;

- $\quad$ Praxeologías, saberes que se utilizan como herramientas -matemáticas o no- para estudiar las distintas respuestas halladas y construir la respuesta a $Q$.

A diferencia de lo que ocurre en la enseñanza monumental, donde es el profesor quien toma todas las decisiones relativas al medio de estudio, en un REI el desarrollo del medio es, además, una responsabilidad de los estudiantes. Ellos pueden incorporar a él cualquier instrumento y herramienta que consideren pertinente y posiblemente útil.

En nuestro caso, el REI se desarrolla a partir de la pregunta generatriz Q: ¿Por qué se cayó la Piedra Movediza (PM) de Tandil?, respecto de la cual se busca elaborar una respuesta científicamente válida. La pregunta engendra un recorrido que involucra el estudio conjunto de saberes matemáticos y físicos, que por razones de espacio no desarrollamos aquí y que se 
sintetizan en la Figura 1. El Modelo Epistemológico de Referencia (MER), esquematizado en la Figura 1, es una reconstrucción de los investigadores sobre los saberes involucrados en el estudio de una cierta cuestión generatriz (Chevallard, 2013a). Un análisis detallado del MER, para este caso, puede encontrarse en Gazzola (2018), Gazzola, Otero, Llanos y Arlego (2018), Otero et al. (2016) y Otero, Llanos y Arlego (2017).

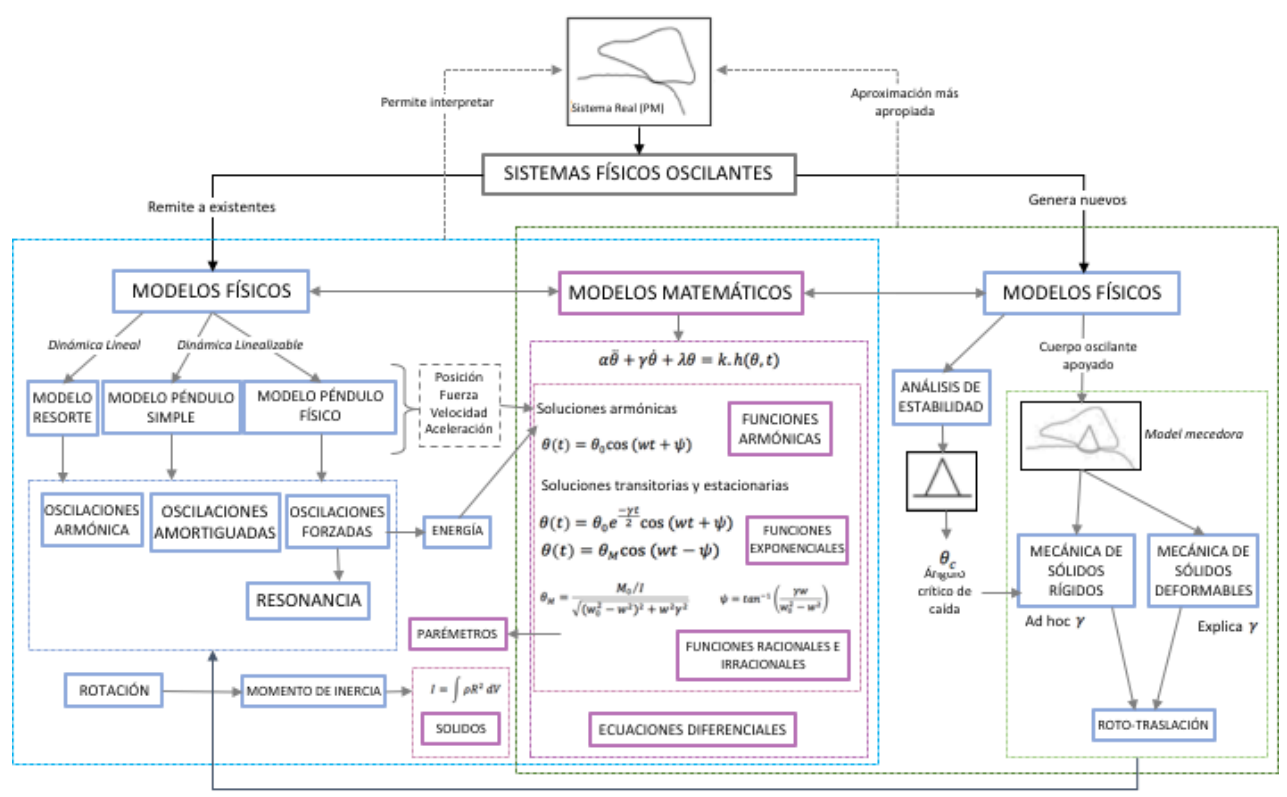

Figura 1: Esquema de síntesis del MER del REl.

Fuente: Elaboración propia.

\subsection{Dialécticas del estudio y la investigación}

La TAD define un conjunto de gestos o acciones didácticas básicas, denominadas dialécticas (Chevallard, 2013a), que son características teóricas de los REI. El término dialéctica alude a dos polos de acciones contrapuestas dentro de un proceso de estudio, que no son duales sino dinámicas y se relacionan y convocan mutuamente. Hasta el momento se han definido nueve dialécticas:

D1. Dialéctica del estudio y la investigación, o de las preguntas y las respuestas: Se refiere a la continua formulación de cuestiones y elaboración de respuestas, que pueden ser tanto orales como escritas. El desarrollo de un REI requiere, por un lado, investigar los saberes que están disponibles en la cultura institucional y que podrían ser apropiados para responder a $Q$ 
abarcando un contexto amplio, y por otro, enfocarse en estudiar de manera pormenorizada ciertos saberes específicos.

D2. Dialéctica media-medio: Se refiere al proceso de validación en un REI. Todo saber es producto de una conjetura y, como tal, debe ser puesto a prueba. Se llama media a los sistemas de información que emiten mensajes a un cierto público, por ejemplo: el curso de un profesor, un programa de televisión, un libro de texto, un sitio en internet, etc. Los sistemas de información pueden volverse parte del medio, por lo tanto este último no está definido de antemano, y es elaborado a medida que se desarrolla el REI. De este modo, la información (media) será incorporada al medio cuando se pruebe su pertinencia y validez para la construcción de respuestas.

D3. Dialéctica del individuo y el colectivo: En un REI, cada actor del sistema didáctico es responsable por estudiar $Q$ y colaborar en la producción de una respuesta conjunta. El tratamiento de una pregunta compleja requiere de un reparto de responsabilidades, para luego evaluar las respuestas obtenidas en la comunidad. En este sentido, el estudio colectivo es muy diferente del llamado "trabajo en grupos", donde todos tratan el mismo asunto al mismo tiempo.

D4. Dialéctica del paracaidista y las trufas: Estos dos términos son metafóricos, y fueron utilizados por el historiador Emmanuel Leroy-Ladurie al hacer referencia a los historiadores a quienes clasificaba como paracaidistas, por un lado, o buscadores de trufas, por el otro. En el primer caso se refiere a la acción de explorar en áreas extensas de territorio, vistas "desde arriba", y en el segundo, a la búsqueda de tesoros enterrados tales como las trufas. El "explorador" toma distancia del problema y observa el terreno desde afuera, pero en algún momento deberá inspeccionarlo desde muy cerca, para encontrar la solución. En un REI ambas acciones son necesarias: inspeccionar grandes áreas de conocimiento hasta avizorar aquel saber que es la "pepita de oro", en este caso de verdad, e ir por él para estudiarlo en detalle.

D5. Dialéctica de entrar y salir del tema: Durante la búsqueda de respuestas a la pregunta generatriz, se incorporan saberes al medio para estudiarlos. Esto puede llevar a "salir de un tema o saber" para encontrar otros y luego regresar nuevamente al tema inicial. Estas entradas y salidas requieren analizar y decidir cuáles saberes se considerarán pertinentes para construir una respuesta.

D6. Dialéctica de las cajas negras y las cajas claras: Una vez identificados los saberes útiles para responder a la pregunta generatriz, es necesario establecer cuánto estudiar de cada uno de ellos 
y encontrar un nivel de gris adecuado. Los saberes relevantes serán "aclarados o clarificados", mientras que los otros se dejarán en la "oscuridad".

D7. Dialéctica de la lectura y la escritura: En un REI es necesario analizar y registrar por escrito la parte útil de las respuestas encontradas, es decir, reescribirlas. Ellas son analizadas y evaluadas para luego interpretarlas con relación al problema que se intenta resolver, elaborando notas de síntesis, glosarios, conclusiones finales, etc.

D8. Dialéctica del análisis (y síntesis) praxeológico y didáctico: Todo análisis praxeológico (de un saber involucrado) requiere formular ciertas preguntas que son de corte didáctico y que se relacionan con su difusión, por ejemplo: ¿De dónde viene este saber? ¿Cómo surge en esta institución?, etc. Por su parte, esto lleva a formular preguntas sobre el saber en sí: ¿En qué consiste el saber?

D9. Dialéctica de la difusión y la recepción: Se refiere a la comunicación y justificación de la respuesta obtenida, la cual es el producto de la actividad matemática desarrollada por la comunidad; en consecuencia, la comunicación debe explicar las respuestas obtenidas y convocar al cuestionamiento y a la aceptación o no. Se trata de una difusión epistémica, preferentemente escrita, en la cual existe una justificación para cada respuesta obtenida, cada saber utilizado o cada camino recorrido en la construcción de una respuesta.

\section{METODOLOGÍA}

Este trabajo tiene como finalidad contribuir a la operacionalización del constructo teórico dialéctica. Las dialécticas resultan sumamente útiles para analizar el desarrollo de un REI en una cierta institución, en este caso, en la escuela secundaria. Si bien la naturaleza de esta investigación es cualitativa porque la didáctica es una ciencia social, empleamos una metodología cualitativa basada en la construcción inductiva de categorías y subcategorías de análisis, que luego son transformadas en variables nominales y modalidades que permiten la utilización de técnica estadísticas cuantitativas multivariadas.

\subsection{Contexto de implementación}

Se realizaron cinco implementaciones del REI en cursos de Matemática de escuelas secundarias en Argentina y participaron en total N=116 estudiantes, de entre 16 y 18 años. El desarrollo del REI significó grandes cambios en la organización de la enseñanza y en la dinámica de las clases. 
De manera general, es posible enunciar las siguientes características comunes a las cinco implementaciones del REI:

- $\quad$ La pregunta $Q$ fue propuesta por el profesor el primer día de cada implementación, con un conjunto de materiales bibliográficos relativos al problema: recortes de diarios periodísticos, textos de divulgación, crónicas historiográficas, extractos de libros.

- $\quad$ El objetivo era elaborar una respuesta a $Q$. Los estudiantes tenían que formular nuevas preguntas, buscar respuestas y estudiar la matemática y la física pertinentes.

Los estudiantes conformaron grupos de estudio de cuatro integrantes, cada uno disponía de al menos una computadora para utilizar applets, physlets y software de matemática o buscar información en internet.

- $\quad$ Los estudiantes podían consultar libros de texto de la biblioteca, además de los que el profesor llevó a las clases. Específicamente, para estudiar los contenidos de Matemática y Física, el profesor propuso dos textos como soporte con tareas asociadas a cada uno de ellos. El profesor fue un sistema de información más.

- $\quad$ Cada encuentro terminó con una puesta en común, con el objetivo de comunicar las respuestas y ponerlas a consideración de la clase.

- $\quad$ Durante el recorrido se realizaron resúmenes parciales y, al final del estudio, una síntesis general que incluyó las preguntas formuladas durante el REl, sus respuestas, los saberes estudiados y la respuesta $Q$ construida.

\subsection{Recolección y análisis de datos}

Se realizó observación participante y no participante y se tomaron notas de campo de cada encuentro. Se recolectaron todos los protocolos escritos de los estudiantes. Al final de cada clase, el profesor-investigador retiraba las producciones escritas de los estudiantes y la síntesis parcial de los grupos. Estos documentos se devolvían en el encuentro siguiente una vez digitalizados.

Inicialmente se realizó una categorización inductiva a partir de los protocolos de los estudiantes por medio de técnicas cualitativas de análisis y metaanálisis. En una segunda etapa, se emplearon técnicas cuantitativas de estadística multivariada como el Análisis Exploratorio de Datos (Lebart \& Morineau, 1994), a partir del cual se transformaron las categorías en un conjunto de variables 
nominales y sus respectivas modalidades. Las variables corresponden a cada dialéctica y las modalidades se refieren a las distintas formas en que la dialéctica se identifica en los protocolos escritos de los estudiantes. Luego, utilizando el software SPAD8.1 ${ }^{\circledR}$ se realizaron un estudio de contingencia y un análisis factorial de correspondencias múltiples.

\subsection{Test de aleatoriedad}

Para despejar la posibilidad de una distribución aleatoria de los datos, atendiendo a que se trata de un estudio basado en un número relativamente pequeño de individuos, se realizó un test de Montecarlo (Otero et al., 2002). Primero, se generaron aleatoriamente 100 matrices (muestras) de datos de 116 filas por 7 columnas, correspondientes a los individuos y a las variables del estudio, respectivamente. Para cada muestra se desarrolló el análisis factorial correspondiente y se retuvieron los tres primeros autovalores, los porcentajes de la varianza explicada por cada uno de ellos y la varianza acumulada por los tres primeros factores. En cada muestra se calculó la media $(\bar{x})$ y la desviación típica $(\sigma)$ del porcentaje total de la varianza explicada y sus valores extremos. Con los valores del porcentaje total de la varianza explicada en las cien muestras aleatorias, se construyó el histograma que fue ajustado por una curva Normal, como se muestra en la Figura 2.

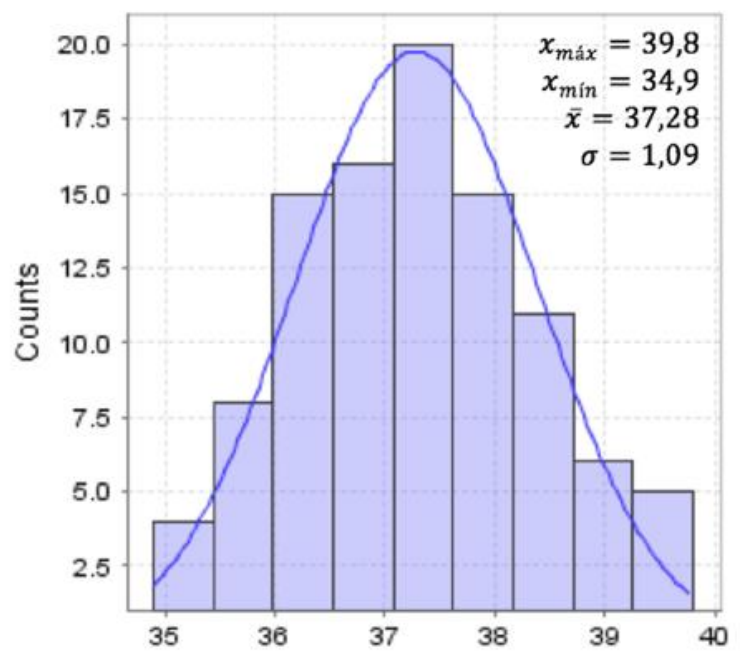

Figura 2: Histograma de frecuencias del porcentaje total de la varianza explicada por los tres primeros factores en 100 muestras aleatorias.

Fuente: Elaboración propia. 
El gráfico muestra que la distribución es aproximadamente normal y que el porcentaje total de la varianza explicada por los tres primeros factores tiene probabilidad casi nula para valores superiores al 40\%. Como en los datos reales, nuestro estudio acumula el 69,62\% de la varianza explicada en los tres primeros factores (Gazzola, 2018), por lo que resulta casi nula la probabilidad de que los datos sean aleatorios $(p<0,0001)$.

\section{CATEGORÍAS DE ANÁLISIS Y VARIABLES NOMINALES}

En la Tabla 1 se sintetizan las variables y sus respectivas modalidades. A continuación, se describe cada una de ellas.

Tabla 1

Variables y modalidades

\begin{tabular}{|c|c|c|}
\hline Variables & Modalidad & Ref. \\
\hline \multirow{2}{*}{ D1 } & No hay búsquedas & D1NB \\
\hline & Búsqueda y estudio de respuestas & D1BE \\
\hline Preguntas y respuestas & Formulación de nuevas preguntas & D1NP \\
\hline \multirow{3}{*}{$\begin{array}{l}\text { D2 } \\
\text { Media-medio }\end{array}$} & No hay D2 & $\mathrm{D} 2 \mathrm{NC}$ \\
\hline & $\begin{array}{l}\text { Se cuestionan resultados obtenidos a partir de saberes propuestos por } \\
\text { el profesor }\end{array}$ & $\mathrm{D} 2 \mathrm{CP}$ \\
\hline & $\begin{array}{l}\text { Estudio y cuestionamiento de otras respuestas distintas a las del } \\
\text { profesor }\end{array}$ & D2ON \\
\hline D3 & No hay D3 & D3NO \\
\hline Individuo y colectivo & Se diferencian las respuestas individuales de las grupales & D3SI \\
\hline \multirow{3}{*}{$\begin{array}{l}\text { D5 } \\
\text { Entrar y salir del tema }\end{array}$} & No hay D5 & D5NO \\
\hline & Salidas y entradas inmotivadas a distintos saberes & D5LO \\
\hline & Salidas y entradas orientadas a distintos saberes y al problema inicial & D5GL \\
\hline D6 & No hay D6 & D6NO \\
\hline \multirow{2}{*}{$\begin{array}{l}\text { Cajas negras y cajas } \\
\text { claras }\end{array}$} & Identificación de saberes pertinentes y funcionales a $Q$ & D6SP \\
\hline & Se determina qué y cuánto estudiar de un saber & D6AL \\
\hline \multirow{3}{*}{$\begin{array}{l}\text { D7 } \\
\text { Lectura y escritura }\end{array}$} & No hay D7 & D7NO \\
\hline & Recopilación de respuestas parciales & D7RP \\
\hline & Lectura, interpretación y reescritura & D7RE \\
\hline \multirow{3}{*}{$\begin{array}{l}\text { D9 } \\
\text { Difusión y recepción }\end{array}$} & No hay difusión & D9NO \\
\hline & Difusión narrativa & D9NA \\
\hline & Difusión epistémica & D9EP \\
\hline
\end{tabular}

Fuente: Elaboración propia. 
D1. Dialéctica de las preguntas y las respuestas. Esta variable presenta tres modalidades:

- D1NB: No hay búsquedas. No se realizan búsquedas en ningún sistema de información, ni se plantean nuevas preguntas fuera de las que propone el profesor o se acuerda estudiar.

- $\quad$ D1BE: Búsquedas y estudio de respuestas. Identifica a los estudiantes que exploran los sistemas de información (recortes de diarios, libros sobre la Piedra Movediza (PM), textos del profesor, internet, libros de la biblioteca, etc.) para construir respuestas posibles o que estudian las ya conocidas (etiquetadas) y/o los saberes útiles para responder a $Q$ o a sus derivadas. Por ejemplo, se estudian:

- $\quad$ Características de la PM y posibles hipótesis de su caída

- Movimiento armónico simple

- Ángulos y razones trigonométricas

- $\quad$ Funciones armónicas

- $\quad$ Energía mecánica

- Movimientos oscilatorios amortiguados y forzados

- Resonancia mecánica

- D1NP: Formulación de nuevas preguntas. Identifica a quienes, además de buscar información y estudiar, proponen nuevas preguntas y estudios, principalmente sobre: (a) saberes matemáticos y/o físicos, (b) la vinculación de dichos saberes con la PM, (c) la PM.

A modo de ejemplo, la Figura 3 muestra el protocolo del estudiante A79, quien buscó en internet y en libros de física de la biblioteca para estudiar el fenómeno de la resonancia. Esto derivó en la formulación de nuevas de preguntas acerca de dicho fenómeno, de los sistemas oscilatorios y su posible vinculación con la PM. 


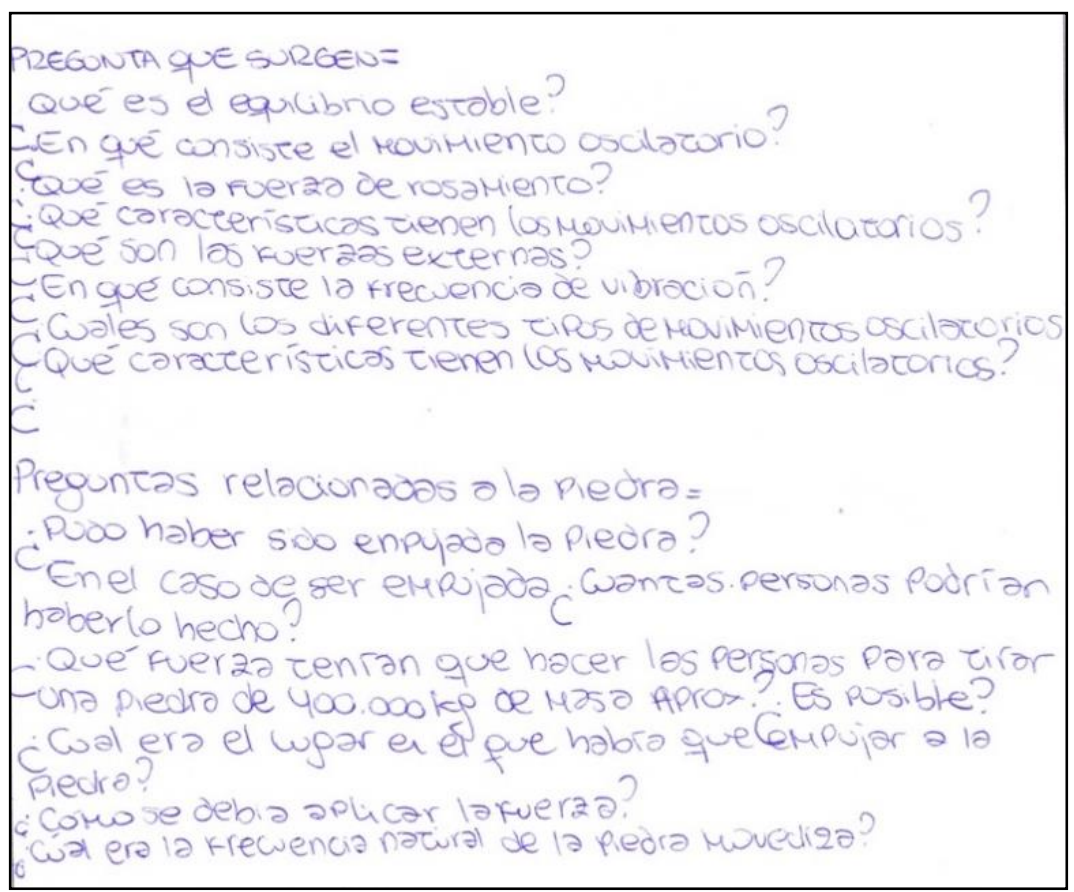

Figura 3: Protocolo correspondiente a A79.

Fuente: Datos de la investigación.

D2. Dialéctica de los medio-media. Considera el tipo de preguntas que los estudiantes formularon, identificando si estas cuestionan algún resultado obtenido de algún sistema de información. Las modalidades encontradas son:

D2NC: No hay D2. No se ponen a prueba los elementos del medio ni se realiza ningún cuestionamiento sobre la validez de las respuestas encontradas, ni de las que propone el profesor. Directamente se aceptan como válidas y se utilizan.

D2CP: Se cuestionan resultados obtenidos a partir de saberes propuestos por el profesor.

Los estudiantes cuestionan la validez y pertinencia de respuestas que ingresan al medio, requiriendo nueva información. Por ejemplo:

- Se formulan preguntas en términos de ¿por qué? o ¿cómo?: ¿'Por qué la PM es un sistema oscilante? ¿Cuál de todos los modelos se ajusta mejor a la PM y por qué? ¿Por qué la PM no realiza un MAS? ¿Cómo probar que la piedra se cayó por resonancia?, etc.

- Se utilizan diferentes recursos y herramientas para validar una respuesta dada. 
D2ON: Estudio y cuestionamiento de otras respuestas distintas a las del profesor. Los estudiantes, además de analizar la pertinencia y validez de las distintas respuestas, consideran o incorporan a $M$ una respuesta o un saber que obtuvieron de algún sistema de información (no el profesor) para ser analizada y estudiada.

Por ejemplo, la Figura 4 corresponde a la respuesta del estudiante A102. Al estudiar la frecuencia de oscilación $(w)$ de un MAS, se propuso una tarea que solicitaba generar un sistema oscilante considerando un $w$ determinado. Hasta el momento solo se había tratado el caso del resorte, pero A102 buscó en internet e investigó sobre posibles sistemas oscilantes y decidió considerar el caso del péndulo y sus características: movimiento, período, ángulo de oscilación, longitud de la cuerda. Este estudiante incorporó al medio esta respuesta, que fue analizada y validada por la clase. También propuso un modelo tipo "mecedora" como el "más adecuado" al sistema de la PM que un péndulo.

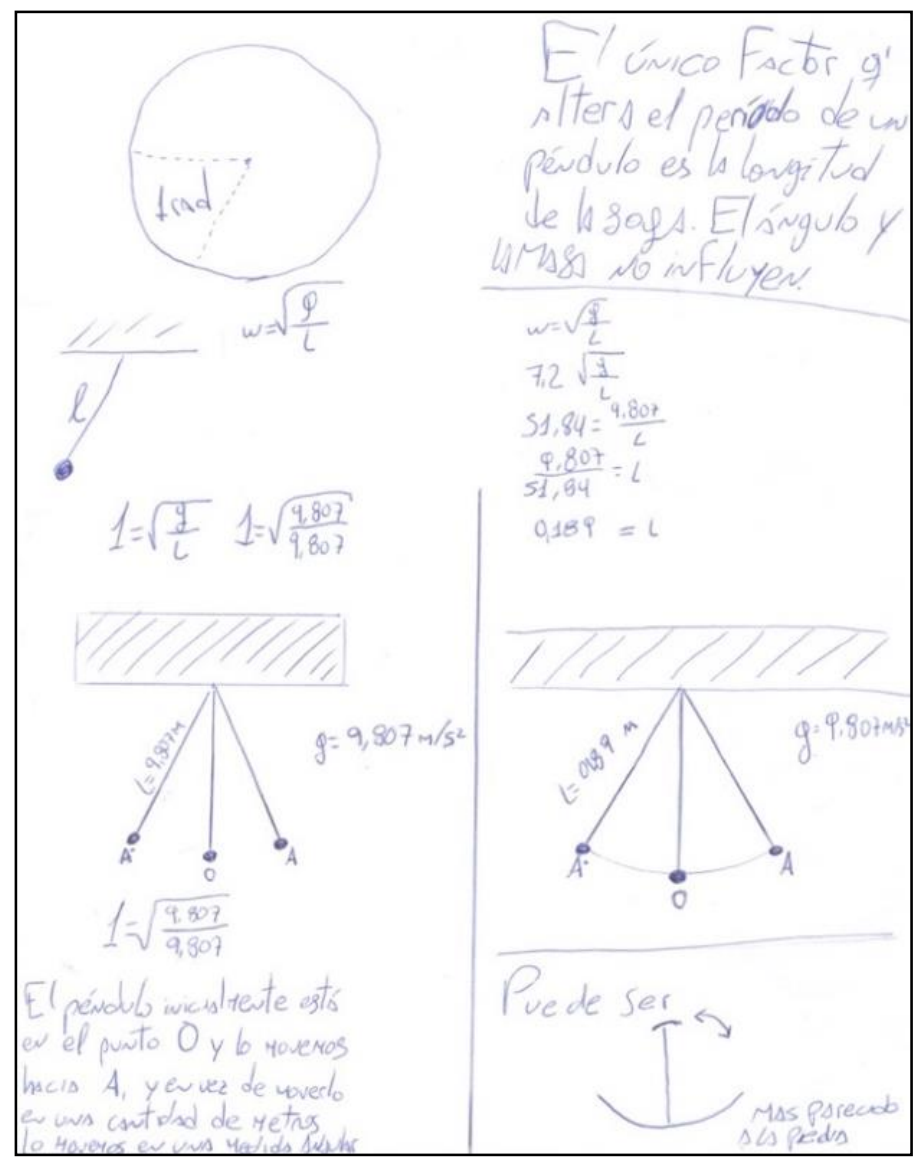

Figura 4: Protocolo correspondiente al estudiante A102.

Fuente: Datos de la investigación. 
D3. Dialéctica del individuo y el colectivo. Se tiene en cuenta si los estudiantes distinguen entre las respuestas elaboradas individual o colectivamente. Tiene dos modalidades:

D3NO: No hay D3. Estudiantes que no distinguen entre las respuestas obtenidas individualmente de aquellas obtenidas del estudio colectivo.

- $\quad$ D3SI: Se diferencian las respuestas individuales de las grupales.

Por ejemplo, la Figura 5 corresponde al protocolo del estudiante A111. En la parte superior, se observa su respuesta individual relativa a las características del movimiento de un resorte horizontal, y luego se observa que él "completó" dicha respuesta a partir del estudio realizado con su grupo. Para diferenciarlas utilizó una línea.

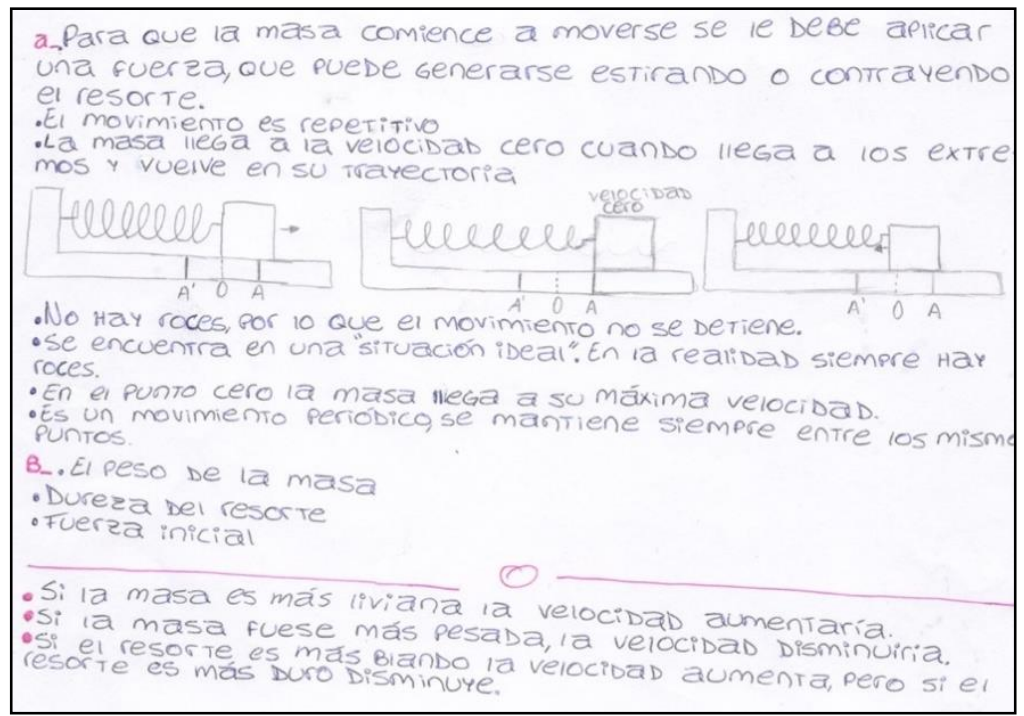

Figura 5: Protocolo correspondiente al estudiante A111.

Fuente: Datos de la investigación.

D5. Dialéctica de entrar y salir del tema. Para aportar una respuesta a $Q$ es necesario "salirse" del problema inicial e ingresar en el estudio de saberes matemáticos y/o físicos, y retomarlo posteriormente. Las modalidades son:

- $\quad$ D5NO: No hay D5. Estudiantes que "salen del tema" pero no regresan a él. Es decir, estudian matemática y física pero no utilizan esos saberes para la construcción de las respuestas. Se identifican, por ejemplo: 
- Salidas hacia la física para el estudio de las oscilaciones.

- Salidas hacia la matemática para el estudio de las funciones trigonométricas.

- Dentro de la matemática o la física, salidas para estudiar otros conocimientos dentro de la disciplina. Por ejemplo en matemática, salir para estudiar ángulos en el plano y razones trigonométricas, o en física, salir para estudiar el concepto de energía mecánica.

D5LO: Salidas y entradas inmotivadas a distintos saberes. Se realizan "salidas" y "entradas" a la matemática y la física de manera local e inmotivada, es decir, sin considerar el problema que dio origen al estudio. Por ejemplo:

- Los resultados obtenidos en una disciplina son interpretados y utilizados para avanzar en el estudio de la otra, aunque no se relacionan con el problema.

- Se utilizan los saberes estudiados, las técnicas construidas o algunos resultados obtenidos dentro de una disciplina para avanzar en el estudio de esa disciplina.

Se propone como ejemplo el protocolo de A113 (Figura 6). Este estudiante "sale" hacia la matemática para estudiar las funciones armónicas y posteriormente "entra" en la física, interpretando la expresión armónica de la posición dependiente del tiempo para un MAS. EI estudiante analiza la función seno, la incidencia de sus parámetros en la representación gráfica y la interpreta para el resorte.

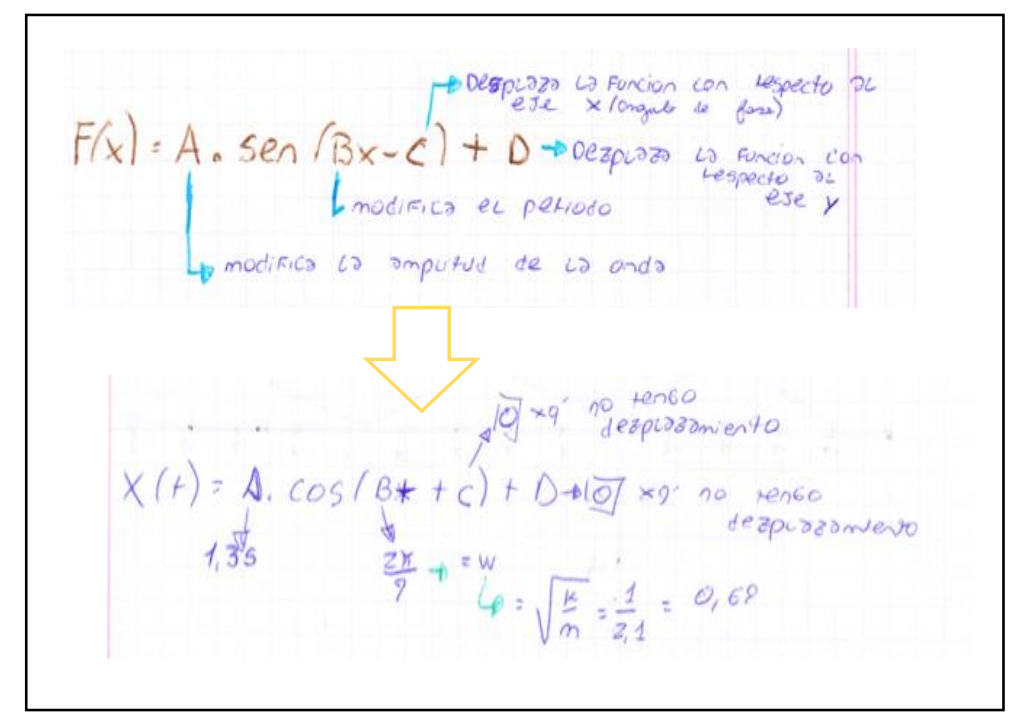

Figura 6: Fragmento de un protocolo correspondiente a A113.

Fuente: Datos de la investigación. 
- D5GL: Salidas y entradas orientadas a distintos saberes y al problema inicial. Se refiere

a los estudiantes que siempre tienen presente al sistema real. Es decir, realizan "salidas y entradas" motivadas por la pregunta generatriz y consideran continuamente aquellos saberes matemáticos y físicos funcionales al fenómeno de la PM. Por ejemplo, estudian:

- $\quad$ El modelo de resorte, y establecen analogías entre su movimiento y la PM.

- $\quad$ Las funciones seno y coseno, y relacionan sus propiedades con el resorte y con la PM.

- Los sistemas oscilantes, y buscan "nuevos sistemas" que se asemejen a la PM, tales como la "mecedora".

- Y readaptan los gráficos posición-tiempo de las oscilaciones amortiguadas y forzadas al movimiento de la PM.

D6. Dialéctica de las cajas negras y las cajas claras. Se considera si los estudiantes determinan qué saberes son pertinentes y merecen ser profundizados, aclarados, etc. y si utilizan lo estrictamente necesario (en un nivel de gris adecuado). Las modalidades son: D6NO: No hay D6. Estudiantes que no identifican cuáles saberes son pertinentes para elaborar la respuesta a $Q$ o sus derivadas. Ellos estudian solo lo que propone el profesor.

- D6SP: Identificación de saberes pertinentes y funcionales a Q. Se establece cuáles conocimientos son necesarios estudiar para avanzar en la construcción de una respuesta. Se recupera solo la parte útil de los saberes estudiados, necesaria para determinada respuesta. Por ejemplo, estudiantes que para entender el movimiento y la caída de la PM deciden estudiar las oscilaciones, o que para describir matemáticamente al MAS establecen que son necesarias las funciones trigonométricas, o que para estudiar los distintos tipos de oscilaciones amortiguadas (sub, sobre y crítica) consideran que es útil estudiar las exponenciales decrecientes.

- $\quad$ D6AL: Se determina qué y cuánto estudiar de un saber. Además de establecer cuáles saberes son pertinentes, se determina el alcance del estudio, o el "nivel de gris". Por ejemplo, se estudian:

Las funciones trigonométricas seno y coseno, y se deja de lado la tangente.

La energía cinética y potencial, considerando la ecuación en función del tiempo. 
- Las condiciones de equilibrio, considerando un triángulo apoyado que gira sobre uno de sus vértices.

Por ejemplo, la Figura 7 presenta el protocolo de A89, quien para calcular el ángulo crítico de oscilación, estudia parcialmente (parte inferior de la figura) las condiciones de equilibrio a partir de un triángulo apoyado, pues solo considera lo que es necesario y útil para elaborar su respuesta.

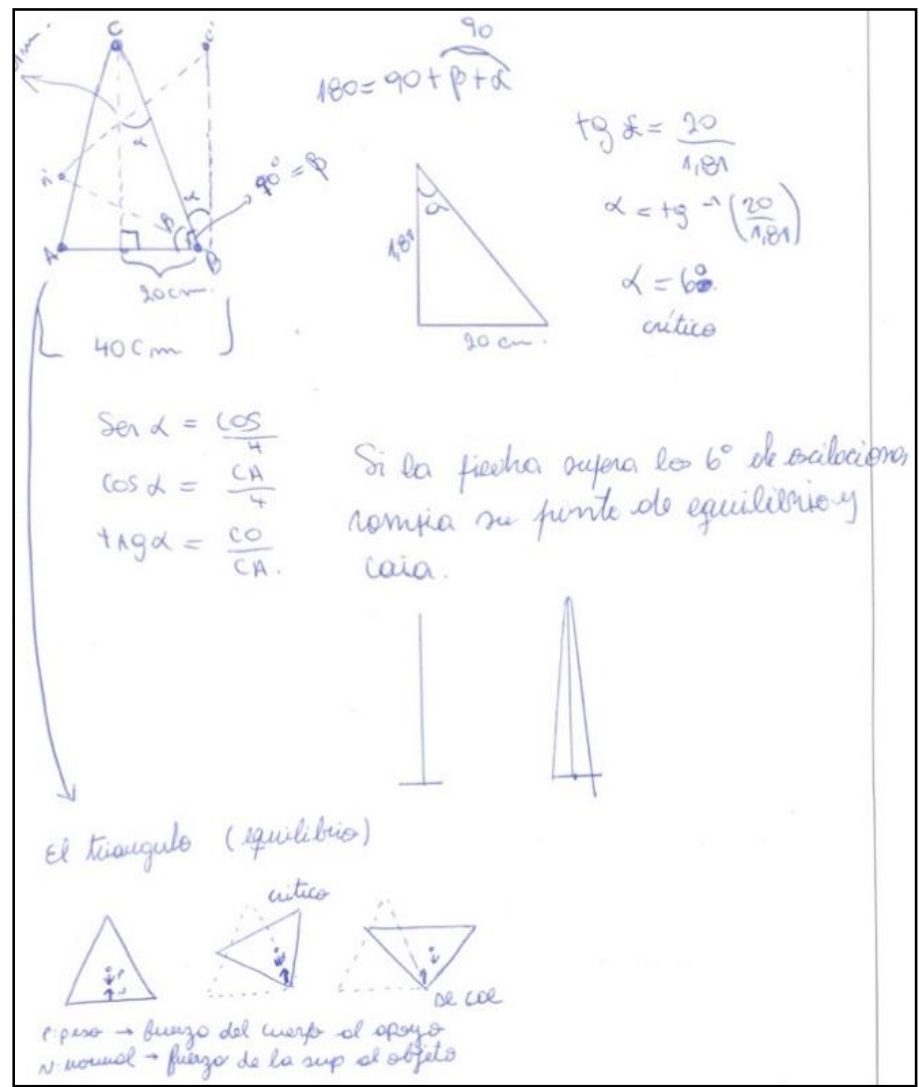

Figura 7: Protocolo correspondiente al estudiante A89.

Fuente: Datos de la investigación.

D7. Dialéctica de la lectura y la escritura. Se considera si los estudiantes analizan, interpretan y reescriben las respuestas encontradas, sin copiar textualmente, rescribiendo la idea que se quiere desarrollar a partir de resúmenes, notas de síntesis, apuntes, etc. Presenta tres modalidades: 
- $\quad$ D7NO: No hay D7. Estudiantes que no leen en distintos sistemas de información ni escriben posteriormente, ni registran la información relevante obtenida de sus lecturas en los documentos sobre la PM, libros de matemática y física, internet, etc.

- $\quad$ D7RP: Recopilación de respuestas parciales. Identifica a quienes realizan las siguientes acciones:

- Marcan, subrayan o resaltan lo que podría ser importante de las búsquedas realizadas en los distintos sistemas de información.

- Reescriben en las carpetas los datos que son útiles para la elaboración de las respuestas o realizan resúmenes de síntesis.

- Anotan del pizarrón, de lo que escribió otro grupo de estudiantes o el profesor.

- Reescriben en las carpetas la información obtenida a partir del uso de algún software como GeoGebra, Mathemática o las planillas de cálculo.

- D7RE: Lectura, interpretación y reescritura. Se interpretan las lecturas y se reescriben, adaptándolas al problema inicial. Por ejemplo:

- Buscar características de la PM y reinterpretarlas a partir de gráficos y esquemas.

- Buscar sobre el fenómeno de resonancia, reinterpretarlo y reescribirlo considerando la PM.

- Realizar lecturas sobre las funciones trigonométricas y reinterpretarlas considerando el movimiento del resorte.

- Realizar una síntesis del propio trabajo, lo que implica releer, volver a interpretar y reescribir los conocimientos estudiados.

La Figura 8 presenta el protocolo de A67. Este estudiante reinterpreta la información encontrada sobre la PM y realiza un dibujo que representa la base de apoyo de la piedra. 


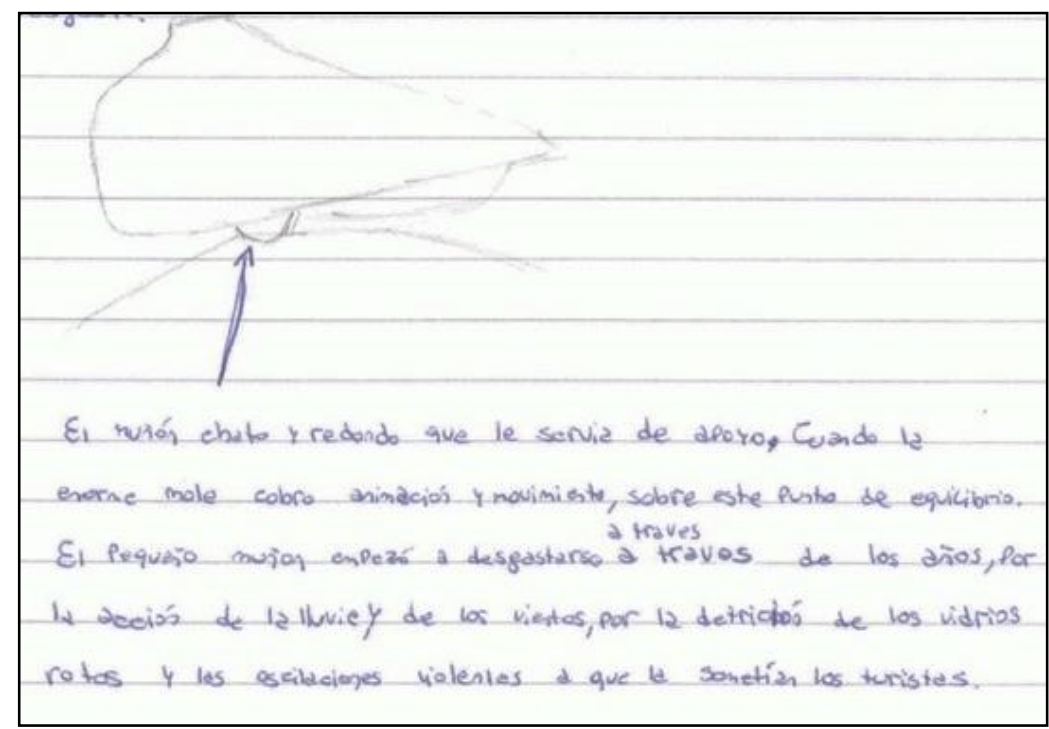

Figura 8: Protocolo correspondiente al estudiante A67.

Fuente: Datos de la investigación.

D9. Dialéctica de la difusión y la recepción. Consiste en la comunicación escrita de las respuestas. Las modalidades son:

- $\quad$ D9NO: No hay difusión. No se realizan síntesis escritas para difundir respuestas.

- D9NA: Difusión narrativa. En este caso se elabora un "relato" que describe las respuestas sin justificar con los saberes estudiados.

- $\quad$ D9EP: Difusión epistémica. Se realiza una difusión fundamentada en saberes. Los estudiantes usan la matemática y la física estudiadas para validar, comunicar y defender sus respuestas. Estas síntesis pueden incluir fórmulas, gráficos, dibujos y esquemas, etc.

La Figura 9 muestra el protocolo de A77. Este estudiante define la resonancia, grafica los sistemas oscilantes considerados (resorte y péndulo) y los tipos de movimientos oscilatorios, estos últimos acompañados de sus fórmulas, para explicar cómo se movía la PM. Además, escribe las funciones trigonométricas utilizadas y la ecuación del movimiento de la piedra. 


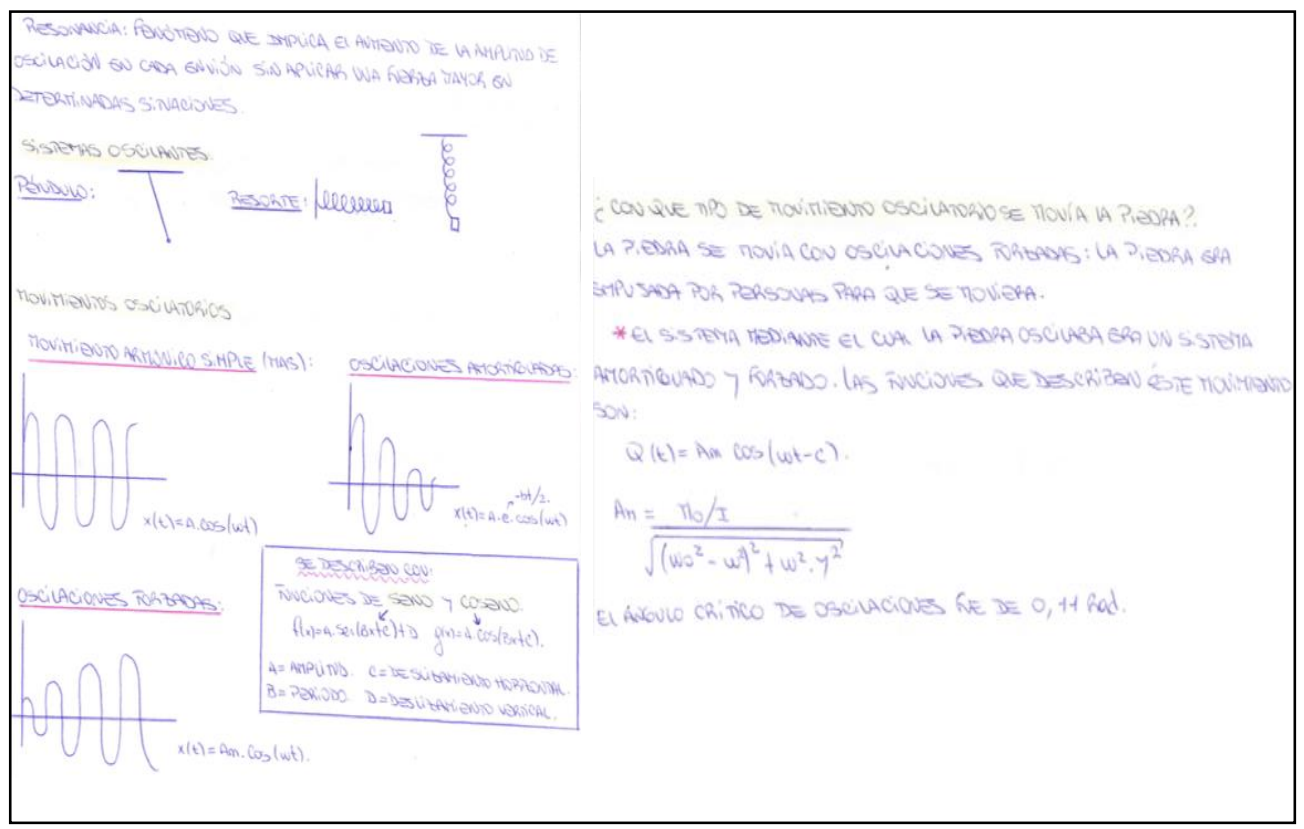

Figura 9: Protocolo correspondiente al estudiante A77.

Fuente: Datos de la investigación.

La dialéctica del paracaidista y las trufas (D4) y la dialéctica del análisis (y síntesis) praxeológico y didáctico (D8) no se pudieron identificar en los protocolos escritos de los estudiantes.

\section{DESCRIPCIÓN DEL FUNCIONAMIENTO DE LAS DIALÉCTICAS}

La Figura 10 presenta el gráfico de frecuencias absolutas de las modalidades asociadas a cada variable. El funcionamiento de las dialécticas lo identificamos a partir de las modalidades representadas con las barras color verde y azul. Las barras de color naranja se refieren a las modalidades que hacen referencia a la ausencia de la dialéctica. Así, teniendo en cuenta estas frecuencias y el total de estudiantes, consideramos como las más frecuentes a las dialécticas de las preguntas y respuestas (D1), de entrar y salir del tema (D5) y de la lectura y escritura (D7). Las dialécticas menos frecuentes son la de media-medio (D2), de las cajas negras y cajas claras (D6), de la difusión y recepción (D9) y del individuo y colectivo (D3). 


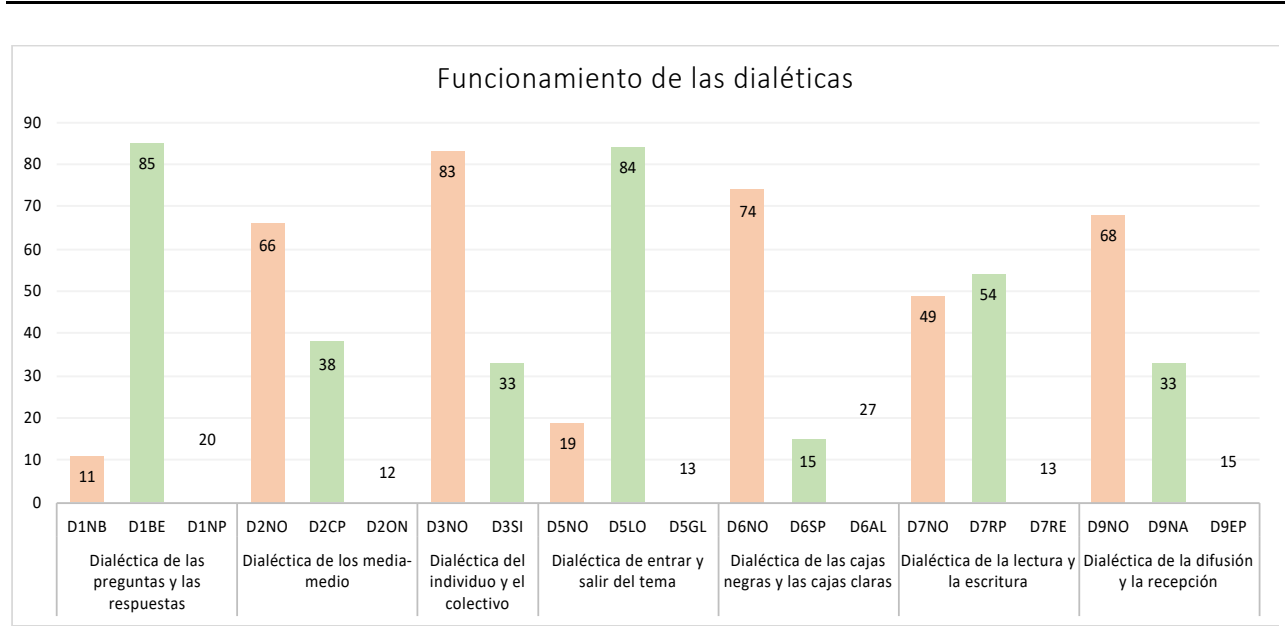

Figura 10: Frecuencia de ocurrencia de las modalidades de cada variable.

Fuente: Elaboración propia.

La dialéctica de las preguntas y las respuestas (D1) se registra en 85 estudiantes que realizan búsquedas y estudios (D1BE). El funcionamiento de esta dialéctica es relativamente débil, ya que solo 20 estudiantes además formulan y proponen nuevas preguntas para estudiar (D1NP).

La dialéctica de entrar y salir del tema (D5) se identifica en 84 estudiantes, aunque de manera inmotivada (D5LO). En este REI los "ingresos y egresos" se producen principalmente entre la matemática y la física, sin retomar el problema inicial. Solo 13 estudiantes consideran esos saberes con relación a la PM (D5GL).

La dialéctica de la lectura y la escritura (D7) se identifica en 67 estudiantes. La mayoría recopila respuestas parciales (D7RP) y muy pocos las leen, interpretan y reescriben (D7RE) con relación a la PM.

La dialéctica media-medio (D2) se identifica en 50 estudiantes, y de ellos 38 se cuestionan sobre la pertinencia y validez de las respuestas (D2CP) que el profesor ingresa al medio y solo 12 incorporan al medio otras respuestas (distintas a las del profesor) que obtienen de sus búsquedas (D2ON).

La dialéctica de la difusión y recepción (D9) se identifica solo en 48 estudiantes. Esto se debe a que las prácticas de escritura son ajenas a la escuela secundaria. Entre los estudiantes que difunden sus respuestas, más de la mitad lo hace de manera narrativa (D9NA), es decir, a partir de un "relato" de las respuestas encontradas, sin basarse en el saber para justificarlas. Solo 15 estudiantes realizan una difusión epistémica (D9EP), fundamentada en dichos saberes. 
La dialéctica de las cajas negras y cajas claras (D6) funciona en total en 42 estudiantes y de ellos, 15 determinan y escriben cuáles saberes son pertinentes y útiles para elaborar las respuestas (D6SP) y 27 deciden cuánto estudiar de ellos (D6AL). Por lo tanto, si bien la frecuencia de esta dialéctica no fue alta, cuando funcionó, mayoritariamente fue para establecer el nivel de gris con el cual estudiar los saberes, tal como propone la TAD.

La dialéctica del individuo y el colectivo (D3) solo se identifica en 33 estudiantes, es decir que, del total de ellos, muy pocos distinguen sus respuestas individuales de las grupales (D3SI). Estos datos revelan además las dificultades para realizar repartos de tareas entre los individuos y los grupos de estudio.

\subsection{Relaciones entre las dialécticas de un REI}

Con el objetivo de establecer posibles relaciones entre las dialécticas, se realizó un análisis de contingencia que evidenció la existencia de asociación estadísticamente significativa $(p<0,001)$ entre ellas. Considerando el coeficiente estadístico V-Cramer, encontramos dialécticas con asociación relativamente fuerte $(0,40$ a 0,60) y fuerte $(0,60$ a 0,80) (Rea \& Parker, 1992), según se detalla en la Tabla 2.

Tabla 2

Variables asociadas

\begin{tabular}{lll}
\hline Variable & Variable asociada & $V$ - \\
& Cramer
\end{tabular}

D1. Dialéctica de las preguntas y las D2. Dialéctica de los media-medio $\quad 0,578$ respuestas

\begin{tabular}{|c|c|c|}
\hline D5. Dialéctica de entrar y salir del tema & D2. Dialéctica de los media-medio & 0,476 \\
\hline D5. Dialéctica de entrar y salir del tema & D7. Dialéctica de la lectura y la escritura & 0,612 \\
\hline $\begin{array}{l}\text { D6. Dialéctica de las cajas negras y las } \\
\text { cajas claras }\end{array}$ & D5. Dialéctica de entrar y salir del tema & 0,488 \\
\hline $\begin{array}{l}\text { D6. Dialéctica de las cajas negras y las } \\
\text { cajas claras }\end{array}$ & D7. Dialéctica de la lectura y la escritura & 0,561 \\
\hline D9. Dialéctica de la difusión y la recepción & $\begin{array}{l}\text { D6. Dialéctica de las cajas negras y las } \\
\text { cajas claras }\end{array}$ & 0,635 \\
\hline D9. Dialéctica de la difusión y la recepción & D7. Dialéctica de la lectura y la escritura & 0,628 \\
\hline
\end{tabular}

Fuente: Elaboración propia. 
Algunas de estas relaciones se presentan aquí por medio de gráficos realizados a partir de la tabla de contingencia de Burt de perfiles horizontales (Crivisqui \& Villamonte, 1997), que permiten analizar cómo se desagregan las modalidades de una variable con respecto a las de la otra. Las Figuras 11 a 14 muestran estos gráficos, que a continuación.

El gráfico de la Figura 11 muestra que los estudiantes que no realizan la dialéctica de entrar y salir del tema (D5NO), tampoco utilizan la de las cajas negras y las cajas claras (D6NO). Entre los estudiantes que realizan entradas y salidas inmotivadas entre los saberes (D5LO), el 67\% no registra funcionamiento de D6, mientras que el $17 \%$ identifica los saberes pertinentes y funcionales a $Q$ (D6SP) y el 16\% determina qué y cuánto estudiar (D6AL). Sin embargo, todos los estudiantes que realizan salidas y entradas orientadas por el estudio de $Q$ (D5GL), también determinan el alcance del estudio (D6AL).

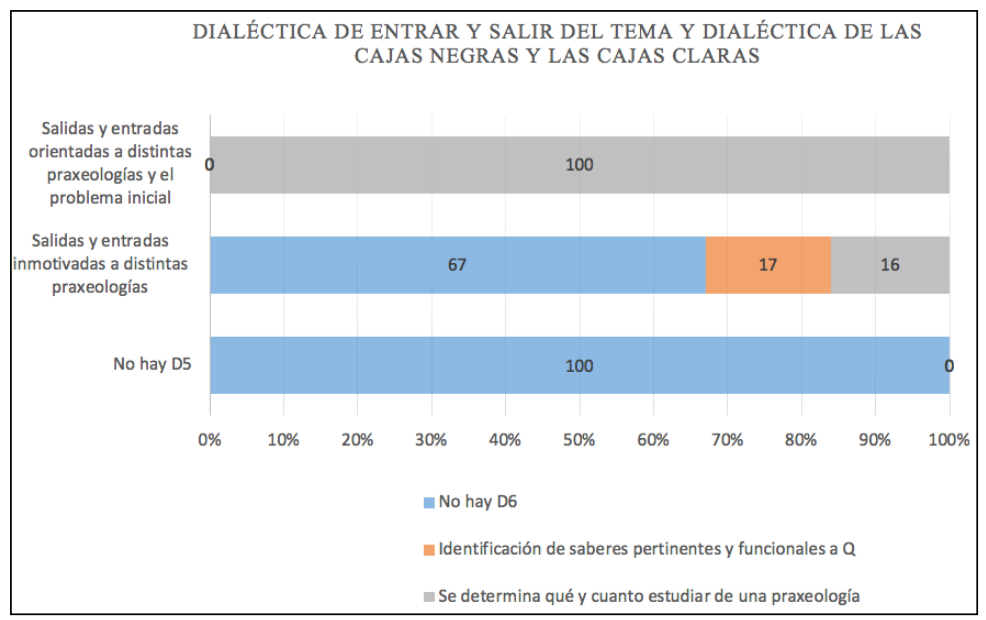

Figura 11: Relación entre D5 y D6.

Fuente: Elaboración propia.

El gráfico de la Figura 12 muestra que los estudiantes que no logran entrar y salir del tema (D5NO), tampoco realizan la dialéctica de la lectura y escritura (D7NO). Entre los estudiantes que ejecutan entradas y salidas inmotivadas entre los saberes (D5LO), el 38\% no produce lecturasescrituras, el 59\% recopila respuestas parciales (D7RP) y solo el 3\% lee, interpreta y reescribe (D7RE). De los estudiantes que entran y salen a los saberes motivados por $Q$ (D5GL), el 23\% recopila respuestas parciales (D7RP) y el 77\% lee, interpreta y reescribe (D7RE). 


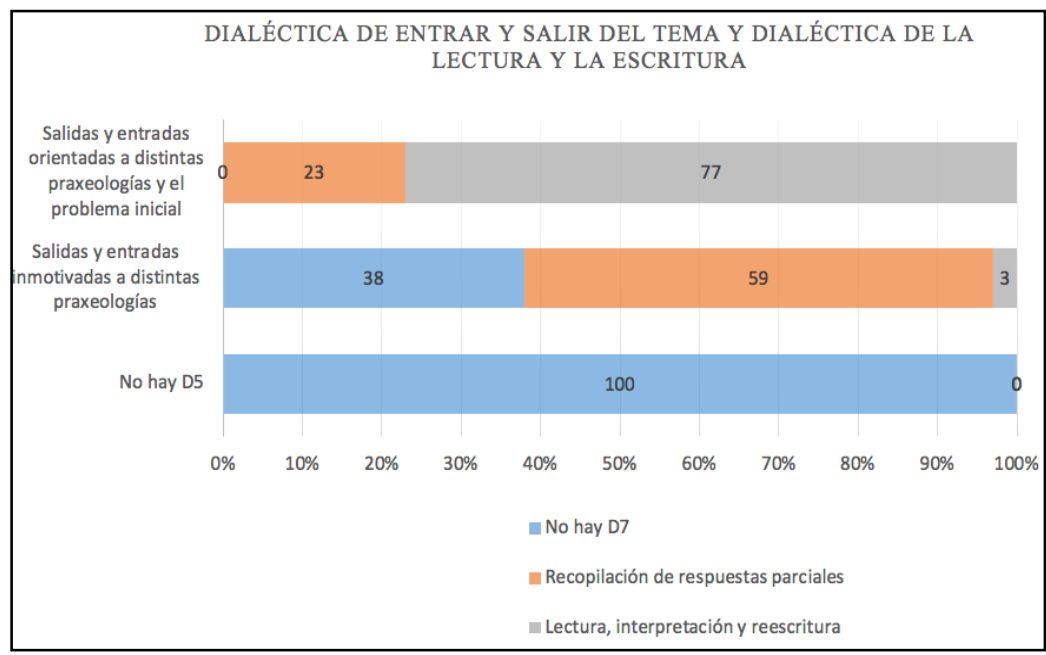

Figura 12: Relación entre D6 y D7.

Fuente: Elaboración propia.

El gráfico de la Figura 13 muestra que entre los estudiantes que no realizan la dialéctica de las cajas negras y las cajas claras (D6NO), el 66\% no ejecuta la de la lectura y la escritura (D7NO) y el 34\% recopila respuestas parciales (D7RP). Entre los estudiantes que identifican los saberes pertinentes y funcionales a $Q$ (D6SP), el 93\% también recopila respuestas parciales (D7RP) y el 7\% las lee, interpreta y reescribe (D7RE). En cambio, entre quienes determinan qué y cuánto estudiar (D6AL), el 56\% recopila respuestas (D7RP) y el $44 \%$ lee, interpreta y reescribe esas respuestas (D7RE).

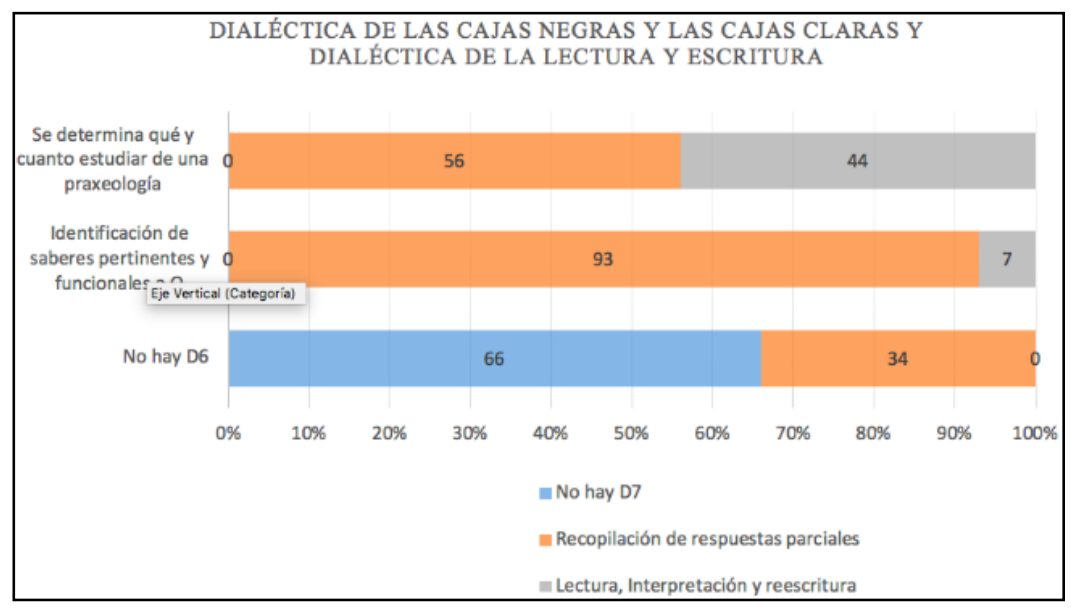

Figura 13: Relación entre D5 y D7.

Fuente: Elaboración propia. 
El gráfico de la Figura 14 muestra que entre los estudiantes que no realizan la dialéctica de la difusión y la recepción (D9NO), el 72\% tampoco ejecuta la de la lectura y la escritura (D7NO), mientras que el $28 \%$ recopila respuestas parciales (D7RP). Entre los estudiantes que realizan una difusión narrativa (D9NA), el 88\% junta saberes parciales y el 12\% los lee, interpreta y reescribe (D7RE). Sin embargo, entre quienes realizan una difusión epistémica (D9EP), el 60\% también lee, interpreta y reescribe (D7RE).

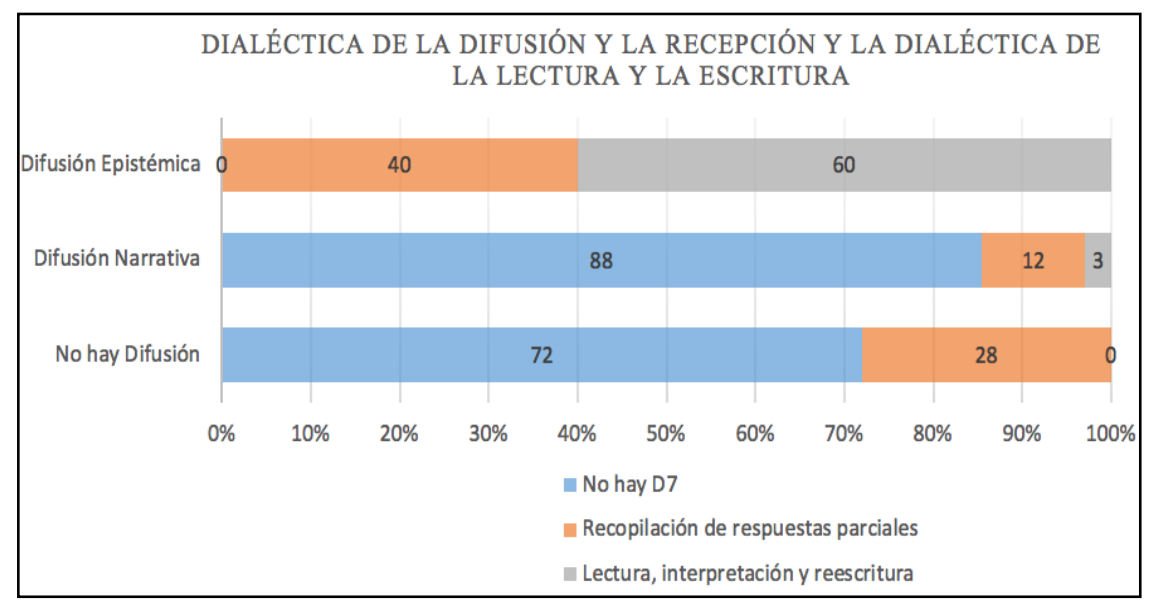

Figura 14: Relación entre D9 y D7.

Fuente: Elaboración propia.

El estudio se completó con un Análisis de Correspondencias Múltiples (ACM) (Benzécri, 1980; Lebart et al., 1985; López-Roldán \& Fachelli, 2015; Moscoloni, 2011) para analizar y caracterizar las similitudes y oposiciones entre las variables cualitativas y sus modalidades. En el ACM los tres primeros factores acumulan el 69,62\% de la varianza explicada. Las variables que más contribuyen a la conformación del primer factor son: dialéctica del individuo y el colectivo (D3), dialéctica de las cajas negras y las cajas claras (D6) y dialéctica de la lectura y la escritura (D7). Esto significa que las mayores diferencias relativas al funcionamiento de las dialécticas en los estudiantes se relacionan con el estudio individual versus el grupal, con la determinación de los saberes pertinentes y el alcance de su estudio y las lecturas, y posteriores escrituras/reescrituras que se realizan.

\section{DISCUSIÓN}

Los datos obtenidos permiten identificar siete de las nueve dialécticas, que funcionan de manera dispar: 
La dialéctica más frecuente es la de las preguntas y las respuestas (D1), lo cual podría atribuirse a las características de una enseñanza por REI, que supone indagaciones constantes para tratar las cuestiones planteadas durante el estudio. Este REI requiere investigar en Matemática y Física y estudiar saberes de ambas disciplinas. Sin embargo, es evidente que la actitud del cuestionamiento es poco habitual y, en consecuencia, es difícil de llevar a cabo por parte de los estudiantes.

La dialéctica de entrar y salir del tema (D5) también se identifica con una frecuencia alta. El estudio de una pregunta en sentido fuerte "obliga" a no limitarse a una sola disciplina y, en este caso, requiere considerar saberes matemáticos y físicos conjuntamente. Sin embargo, la deconstrucción y reconstrucción de estos saberes y su adaptación al problema estudiado, resultó difícil.

La dialéctica de la lectura y la escritura (D7) también tiene una frecuencia relativamente alta. Este resultado podría atribuirse a las diversas búsquedas que los estudiantes realizan y a que el profesor retire diariamente los protocolos escritos, lo cual les exige documentar su trabajo, analizar la información y decidir qué reescribir y cómo. Se observa que las escrituras realizadas son mayoritariamente narrativas y esto está lejos de lo que teóricamente se espera en una clase de Matemática. Sin embargo, las prácticas de lectura y escritura son ajenas a la pedagogía dominante, por lo tanto, llevarla a cabo (aun de manera incipiente) es un aspecto por destacar.

La baja frecuencia identificada en la dialéctica media-medio (D2) podría deberse a que los estudiantes están habituados al monumentalismo y son formados en él, siendo el profesor quien decide qué y cómo estudiar, mientras que ellos "aceptan" los elementos que ingresan al medio sin cuestionar su pertinencia y validez. Entonces, aquí el profesor debió intervenir más de lo deseable para avanzar en el estudio y sus responsabilidades fueron mucho mayores de lo que teóricamente un REI supone. De todas maneras, resulta auspicioso que, al menos algunos estudiantes, pudieron "romper" parcialmente la unilateralidad en la construcción del medio y propusieron estudiar de nuevos saberes que permitieron avanzar en la elaboración de una respuesta a $Q$.

La frecuencia de la dialéctica de las cajas negras y las cajas claras (D6) es igualmente baja y también podría relacionarse con el monumentalismo dominante, ya que tradicionalmente es el profesor quien establece cuáles son los saberes pertinentes y decide cuánto estudiar de ellos para responder a las preguntas planteadas, si es que se plantean. 
La dialéctica de la difusión y la recepción (D9) tiene una frecuencia baja. Cuando ocurre consiste mayoritariamente en una difusión narrativa, que evaluamos como monumental, porque el saber solo se describe, no se usa, y contar la matemática o la física no es hacer matemática ni física. Sin embargo, esta dialéctica implica comunicar las respuestas de manera escrita, y esta acción supone una modificación de gran porte en la cultura escolar, ya que es muy difícil de lograr y requiere de un largo proceso. Por lo tanto, destacamos el hábito de comunicar las respuestas adquiridas en el REl, aunque no haya sido de la manera más apropiada.

Por último, la dialéctica con menor frecuencia es la del individuo y el colectivo (D3). El reparto de tareas y responsabilidades que permiten contribuir a la construcción de una respuesta colectiva, consensuada en y por la clase, es un gesto muy alejado de la enseñanza monumental actual. Los estudiantes están habituados a trabajar individualmente o en pequeños grupos, realizando todos las mismas tareas; romper con este hábito es muy difícil. Consideramos auspicioso que, durante las implementaciones realizadas, algunos pocos estudiantes lograron modificarlo parcialmente.

\section{CONCLUSIÓN}

En este trabajo se utiliza el constructo teórico llamado dialéctica para caracterizar el desarrollo de un REI que involucra a la matemática y a la física, en la escuela secundaria. El uso de las dialécticas como instrumento de análisis ha sido poco explorado en la comunidad de investigadores en la TAD. Su valor reside en que permite evaluar el desarrollo de un REI, así como analizar las principales dificultades y condiciones para su funcionamiento. En este trabajo se mostró cómo operacionalizar este constructo mediante técnicas de análisis cualitativas, como la construcción de una categorización inductiva, y cuantitativas, de análisis estadístico multivariado. Estas últimas permiten identificar cuáles dialécticas ocurren con mayor frecuencia y si ellas están o no correlacionadas.

El desafío de utilizar un dispositivo didáctico propio del paradigma de enseñanza llamado del cuestionamiento del mundo en instituciones donde reina el monumental, produce adaptaciones que modifican sus características. Sin embargo, aun en un estadio germinal se producen algunos gestos propios del cuestionamiento del mundo que conducen a estudiar matemática y física con sentido para los estudiantes. Es una cuestión abierta identificar si el funcionamiento de una cierta dialéctica produce el funcionamiento de otra, aunque se hallaron indicios de una ocurrencia conjunta. 


\section{REFERENCIAS BIBLIOGRÁFICAS}

Barquero, B. (2009). Ecología de la Modelización Matemática en la enseñanza universitaria de las Matemáticas (Tesis doctoral). Recuperado desde https://www.tesisenred.net/handle/10803/3110

Benzécri, J. P. (1980). Practique de l'Anályse des Donneés T 1 y 2. Paris: Dunod.

Chevallard, Y. (2007). Passé et présent de la théorie anthropologique du didactique. Recuperado desde http://yves.chevallard.free.fr/

Chevallard, Y. (2009). La notion de PER: problèmes et avancées. Recuperado desde http://yves.chevallard.free.fr/

Chevallard, Y. (2013a). Éléments de didactique du développement durable. Leçon 1. Enquête codisciplinaire \& EDD. Recuperado desde http://yves.chevallard.free.fr/

Chevallard, Y. (2013b). Enseñar matemáticas en la sociedad de mañana: Alegato a favor de un contraparadigma emergente. Journal of Research in Mathematics Education, 2(2), 161182.

Costa, V. A., Arlego, M., \& Otero, M. R. (2014). Enseñanza del Cálculo Vectorial en la Universidad: propuesta de Recorridos de Estudio e Investigación. Revista de Formación e Innovación Educativa Universitaria, 7(1), 20-40.

Crivisqui E., \& Villamonte G. (1997). Presentación de los métodos de análisis factorial de correspondencias múltiples. PRESTA Programme de recherche et $d^{\prime}$ enseignement en statistique apliqueé, Bruxelles, Belgique.

Donvito, A., Otero, M. R., \& Sureda, P. (2014). Actitudes de la pedagogía de la investigación en el marco de la TAD: un análisis en tres escuelas secundarias. Ikastorratza, 12, 1-27.

Gazzola, M. P. (2018). Diseño, implementación y análisis de un recorrido de estudio e Investigación codisciplinar en matemática y física en la escuela secundaria (Tesis doctoral). Recuperado desde https://www.ridaa.unicen.edu.ar/xmlui/handle/12$3456789 / 2228$

Gazzola, M. P., Llanos, V. C., \& Otero, M. R. (2013). Research and Study Paths in the Teaching of Mathematics at Secondary school relative to the Rational Functions. Journal of Arts \& Humanities, 2(3), 109-115. http://dx.doi.org/10.18533/journal.v2i3.89

Gazzola, M. P., Otero, M. R., Llanos, V. C., \& Arlego, M. (2015). Enseñanza co-disciplinar a la Física y la Matemática en la Escuela Secundaria por medio de Recorridos de Estudio y de Investigación. Revista de Enseñanza de la física, 27(número extra), 117-124. 
Gazzola, M. P., Otero, M. R., Llanos, V. C., \& Arlego, M. (2018). Introducing STEM pedagogy in secondary school by means of the Study and Research Path (SRP). Latin-American Journal of Physics Education (LAJPE), 12(4), 43021-43029. Recuperado desde http://www.lajpe.org/dec18/12_4_02.pdf

Lebart, L., \& Morineau, A. (1994). Systéme portable d'Anályse des Données Numeriques. Saint Mandé: CISIA.

Lebart, L., Morineau, A., \& Fenelon, J. P. (1985). Tratamiento Estadístico de Datos. Barcelona: Marcombo.

Llanos, V. C., \& Otero, M. R. (2013). La pédagogie de l'enquête et du questionnement du monde: une étude longitudinale dans l'école secondaire Argentine. International Journal of Science and Mathematics Education, 7(1), 27-46.

Llanos, V. C., Otero, M. R., \& Gazzola, M. P. (2017). Étude des fonctions algébriques dans le cadre d'un Parcours d'Étude et de Recherche (PER) dans l'école secondaire: le cas des fonctions rationnelles. Educational Journal of the University of Patras UNESCO Chair, 4(1), 1-16. https://doi.org/10.26220/une.2780

López-Roldán, P., \& Fachelli, S. (2015). Análisis factorial. En P. López-Roldán, \& S. Fachelli (Eds.). Metodología de la Investigación Social Cuantitativa, capítulo III.11. Bellaterra: Universitat Autónoma de Barcelona.

Moscoloni, N. (2011). Las Nubes de Datos. Métodos para analizar la complejidad. Rosario: UNR Editora.

Otero, M. R., Fanaro, M., Corica, A., Llanos, V. C., Sureda, P., \& Parra, V. (2013). La Teoría

Antropológica de lo Didáctico en el Aula de Matemática. Tandil: Dunken.

Otero, M. R., Gazzola, M. P., Llanos, V. C., \& Arlego, M. (2016). Co-disciplinary Physics and Mathematics Study and Research Course (SRC) within three study groups: teachers-intraining, secondary school students and researchers. Review of Science, Mathematics and ICT Education, 10(2), 55-78. https://doi.org/10.26220/rev.2315

Otero, M. R., Llanos, V. C., \& Arlego, M. (2017). Development of research and study paths in the pre-service teacher education. European Journal of Educations Studies, 3(8), 214-240. doi:10.5281/zenodo.865887

Otero, M. R., Llanos, V. C., Parra, V., \& Sureda, P. (2014). Pedagogy of Research and Questioning the World: teaching through Research and Study Paths (RSP) in secondary school. Review of science, mathematics and ICT education, 8(1), 7-3. https://doi.org/10.26220/rev.2174 
Otero, M. R., Moreira, M. A., \& Greca, M. I. (2002). El uso de imágenes en textos de física para la enseñanza secundaria y universitaria. Investigações em Ensino de Ciências, 7(2), 127154.

Parra, V., \& Otero, M. R. (2017). Enseñanza de la matemática por recorridos de estudio e investigación: indicadores didáctico-matemáticos de las "dialécticas". Educación Matemática, 29(3), 9-50. doi:10.24844/EM2903.01

Parra, V., \& Otero, M. R. (2018). Antecedentes de los Recorridos de Estudio e Investigación (REI): características y génesis. Revista Electrónica de Investigación en Educación en Ciencias, 13(2), 1-18.

Parra, V., Otero, M. R., \& Fanaro, M. (2013). Los Recorridos de Estudio e Investigación en la escuela secundaria: resultados de una implementación. Bolema, 27(47), 847-874. http://dx.doi.org/10.1590/S0103-636X2013000400008

Rea, L. M., \& Parker, R. A. (1992). Designing and conducting survey research. San Francisco: Jossey-Boss.

Ruiz, N., Bosch, M., \& Gascón, J. (2007). Modelización funcional con parámetros en un taller de matemáticas con Wiris. En L. Ruiz Higueras, A. Estepa, \& F. J. García (Eds.). Sociedad, escuela y matemáticas. Aportaciones de la teoría antropológica de lo didáctico (pp. 677704). Jaén: Publicaciones de la Universidad de Jaén.

Sala, G., Barquero, B., \& Font, V. (2018). Modelización e indagación en la propuesta de un REI codisciplinar de matemáticas e historia. 6éme Congrès International sur la Théorie Anthropologique du Didactique. Congreso llevado a cabo en la Université de Grenoble, Autrans.

Salgado, D., Otero, M. R., \& Parra, V. (2017). Gestos didácticos en el desarrollo de un recorrido de estudio e investigación en el nivel universitario relativo al Cálculo: el funcionamiento de las dialécticas. Perspectiva Educacional. Formación de profesores, 56(1), 84-108. http://dx.doi.org/10.4151/07189729-Vol.56-Iss.1-Art.470 